Anaesthesist 2009 · 58:341-342

DOI 10.1007/s00101-009-1520-5

Online publiziert: 25. März 2009

๑) Springer Medizin Verlag 2009

\author{
R. Zander ${ }^{1} \cdot$ K. Engelhard ${ }^{2} \cdot$ C. Werner ${ }^{2}$ \\ ${ }^{1}$ Physioklin, Mainz \\ ${ }^{2}$ Klinik für Anästhesiologie, Universität Mainz
}

\title{
Volumen macht Druck
}

\section{Kraniell, thorakal, vasal und abdominell}

„Volumen macht Druck“ ist eine Feststellung, die auf alle Kompartimente des Menschen wie Schädel, Thorax, Gefäße und Abdomen zutrifft. Positiv interpretiert, kann sie eine Forderung an den Arzt sein, über einen normalen Volumenstatus einen ausreichenden arteriellen Blutdruck (intravasal) zur Sicherstellung der Organdurchblutung einzustellen. Negativ ist diese Feststellung auch korrekt, wenn eine Volumenzunahme eine unerwünschte Drucksteigerung zur Folge hat, also in der Schädelhöhle (intrakraniell) und im Abdomen (intraabdominell). Innerhalb des Thorax gilt „Volumen macht Druck " für die Blutseite eher negativ, weil der intrathorakale Kreislauf zum Niederdrucksystem gehörig auf niedrige Drücke (und große Volumina) eingestellt ist. Für die Gasseite hingegen, also die Ventilation, würde eher gelten „Druck macht Volumen“: Ein negativer intrathorakaler Druck bewirkt eine physiologische Inspiration und bei künstlicher Beatmung erzwingt ein pathophysiologischer Überdruck eine Insufflation. Insofern sind die Verhältnisse intrathorakal anders, zumal eine gegenseitige Beeinflussung möglich ist: Der intraalveoläre Druck beeinflusst den intravasalen Druck [Valsalva-Manöver, „positive end-expiratory pressure“ (PEEP)] und dieser umgekehrt den intraalvelolären Druck (Lungenödem).

Die Druckmessung ist ein Routinevorgang mit wichtigen Teilaspekten, leider häufig vernachlässigt, von denen mindestens einer entscheidend ist. Beispielsweise wird der vom linken Herzen erzeugte Druck mit einer Manschette am Oberarm als systolischer Druck nur dann korrekt mit $120 \mathrm{mmHg}$ bestimmt, wenn die Man- schette auf die Höhe des Herzens justiert wurde. Damit wird der additive hydrostatische Druck sicher eliminiert. Liegt die Manschette nur 13,6 cm über Herzniveau, wird bereits ein falscher Wert von nur $110 \mathrm{mmHg}$ gemessen, weil oberhalb des Herzniveaus ein negativer hydrostatischer Druck wirksam wird. Da die Dehnbarkeit („compliance“) des Niederdrucksystems (Venen des Körperkreislaufs plus gesamter Lungenkreislauf) deutlich größer ist und die Drücke sehr viel kleiner sind, machen sich hier entsprechende Lageänderungen sichtlich bemerkbar: Die Venen des Handrückens sind bei herabhängendem Arm prall gefüllt, beim Arm nur 13,6 cm oberhalb des Herzniveaus gelegen, werden sie schnell vollkommen entleert.

Dies macht klar, warum bei der Messung des zentralen Venendrucks mit normal nur 4-6 mmHg der Druckaufnehmer möglichst genau auf der Höhe der Katheterspitze (der Ort der Druckmessung) zu justieren ist: Eine Höhenabweichung von nur $7 \mathrm{~cm}$ nach unten verdoppelt den zentralen Venendruck.

Es besteht Einvernehmen, dass nicht der lokale Druck, sondern die Druckdifferenz im entsprechenden Kompartiment die treibende Kraft für die Durchblutung ist, also z. B. der zerebrale Perfusionsdruck („cerebral perfusion pressure“, CPP) für das Gehirn oder der abdominelle Perfusionsdruck („abdominal perfusion pressure“, APP) für die Organe im Abdomen (Darm und Leber). Dazu ist aber erforderlich, dass eine Vereinbarung darüber besteht, an welcher Stelle der zugehörige Blutdruck zu messen ist: Im Fall des CPP auf Höhe des äußeren Gehörgangs, im Fall des APP auf der hydro- statischen Mitte des Abdomens. Dieses Vorgehen stellt sicher, dass Lageveränderungen des Patienten keinen Einfluss auf die Messwerte haben.

Großer Dank gilt den Autoren, die sich in mehrfacher Hinsicht einem Zwang zur Abstimmung unterworfen haben. Alle Drücke werden in mm$\mathrm{Hg}$ angegeben, um auch die Vergleichbarkeit der Atmungsdrücke, z. B. PEEP, mit den anderen Drücken zu erleichtern, auch wenn dies gegen internationale Gepflogenheiten verstößt (PEEP in cm$\mathrm{H}_{2} \mathrm{O}$, mbar; $1 \mathrm{mmHg}=1,36 \mathrm{cmH}_{2} \mathrm{O}$ bzw. $1 \mathrm{cmH}_{2} \mathrm{O}=0,735 \mathrm{mmHg}$ ). Als Symbol für den hydrostatischen Druck wird "p“ verwendet, zugleich englisch „pressure“, in aus dem Englischen unverändert übernommenen Abkürzungen „P“, also intrakranieller Druck (,intracranial pressure“, ICP), zerebraler Perfusionsdruck (CPP), positiver endexspiratorischer Druck (PEEP), systolischer, diastolischer und mittlerer arterieller Druck [„systolic arterial pressure“ (SAP), „diastolic arterial pressure “ (DAP), „mean arterial pressure“ (MAP)], zentraler Venendruck („central venous pressure“, CVP) und intraabdomineller Druck (,intra-abdominal pressure“, IAP) bzw. abdomineller Perfusionsdruck (APP).

Der Zusammenhang zwischen Druck und Volumen wurde von den Autoren auch einheitlich als resultierender Druck (Ordinate) über dem vorgegebenen Volumen (Abszisse) dargestellt, auch wenn die Steigung der Funktion dann die eher ungewöhnliche „elastance“ $(\Delta \mathrm{P} / \Delta \mathrm{V})$ ergibt. Formal aber wird bei Vorgabe einer Volumenänderung und Registrierung des entstehenden Druckes die Elastance be- 
stimmt, z. B. bei der Titration, also Prüfung der Volumenreagibilität, des ICP oder des CVP. Durch einfache Umformung in den Kehrwert wird dann aus der Elastance die Compliance $(\Delta \mathrm{V} / \Delta \mathrm{P})$ erhalten, weil die Dehnbarkeit leichter verständlich scheint.

Die besondere Attraktivität dieser $\mathrm{Pu}$ blikationsreihe besteht in der Tatsache, dass eine anatomische und daher funktionelle Verbindung zwischen den verschiedenen Kompartimenten besteht, die von den Autoren berücksichtigt wurden.

Beispielsweise führt eine Hyperhydratation nicht nur zu einem intravasalen, sondern auch $\mathrm{zu}$ einem intraabdominellen Druckanstieg und dieser seinerseits zu einem intrathorakalen plus intrakraniellen Druckanstieg; ein PEEP von der einen und ein Kapnoperitoneum von der anderen Seite nehmen Einfluss auf den intrathorakalen Blutdruck und damit das Herzzeitvolumen.

Intrakraniell stellen sich die Verhältnisse besonders dar, weil die starre Schädelkalotte keine Volumenänderung zulässt. In dem Beitrag zur „Physiologie und Pathophysiologie der intrakraniellen Druck-Volumen-Beziehung “von Zweckberger et al. wird dieses Problem ausführlich dargestellt: Jede Volumenänderung eines der drei Teilkompartimente Gehirn, Blut und Liquor muss isovolämisch von einem anderen Kompartiment kompensiert werden. Die Bezeichnung Compliance des Gehirns ist in diesem Zusammenhang verwirrend, weil die Dehnbarkeit des inkompressiblen Gehirns formal o $\mathrm{ml} / \mathrm{mmHg}$ betragen muss. Wenn als Normalwert ein Betrag von 0,5-1 ml/mm$\mathrm{Hg}$ genannt wird, bringt dies zum Ausdruck, dass schon eine Volumenzunahme von nur $10 \mathrm{ml}$ zu einer bedenklichen Verdoppelung des ICP führen kann, weil die Volumenverschiebung einen Druckanstieg erzeugt.

Heute wird das Monitoring des Hirndrucks, d. h. die Messung des ICP, als Standard-Monitoring-Verfahren in der Neurointensivmedizin bezeichnet, die Details der Messung sind im Beitrag von Rickels nachzulesen. Da leider immer noch sehr viele Infusionslösungen hypoton sind und damit bei Zufuhr größerer Volumina die Gefahr des Hirnödems mit sich bringen, wird von Zander ein Zusammenhang zwi- schen dem „intrakraniellen Druck und hypotonen Infusionslösungen " hergestellt. Dieses Problem gilt es insbesondere in der Pädiatrie zu vermeiden.

Intrathorakal sind die Verhältnisse dadurch charakterisiert, dass große Volumina kompressiblen Gases (Alveolarraum) in Kontakt zum inkompressiblen Blut (Lungenkreislauf) treten. Die „Beatmung und Volumentherapie beim akuten Lungenversagen" macht dies besonders deutlich, weil die „Auswirkungen auf Gasaustausch und Hämodynamik“ erhebliche klinische Relevanz besitzen, wie von Bercker et al. beschrieben.

Intravasal führt jede Volumenänderung zu sehr unterschiedlichen Druckänderungen, je nachdem ob das Hochdruckoder das Niederdrucksystem betrachtet wird: Ein MAP von ca. $100 \mathrm{mmHg}$ und einem Blutvolumenanteil von nur 15\% im arteriellen System steht einem CVP von nur ca. 5 mmHg mit einem Blutvolumenanteil von ca. $85 \%$ gegenüber. Daraus würde sich eine Compliance-Relation $(\Delta \mathrm{V} /$ $\Delta \mathrm{P}$ ) von 1:50 ergeben, die Literaturangaben hierzu schwanken aber.

Bezüglich der Frage, wie der Volumenstatus eines Patienten möglichst einfach und genau zu erfassen ist, haben sich in den letzten Jahren prinzipielle Unterschiede ergeben: Entweder mit statischen Größen im Niederdrucksystem vor dem rechten Herzen (CVP) bzw. im Hochdrucksystem (MAP) nach dem linken Herzen oder über eine dynamische Antwort auf einen kolloidalen Volumenbolus vor das rechte Herz als sog. Volumenreagibilität („fluid responsiveness“) auf der arteriellen Seite.

Ein dynamisches Verfahren, nämlich den Volumenstatus über die arterielle Pulskurve zu detektieren wird von Pestel et al. vorgestellt. Die korrekte Messung des zentralen Venendrucks als statischer Größe wird von Schummer sehr genau beschrieben:

Essenziell sind die Nullpunktbestimmung, die Kalibration des „transducer“ und die Wahl des richtigen Messzeitpunkts, nämlich endexspiratorisch.

Die Annahme, dass der zentrale Venendruck geeignet ist die kardiale Vorlast zu beurteilen wird von Weyland et al. im nächsten Heft erheblich infrage gestellt. Das Gleiche gilt für eine eindeutige Kor- relation zwischen zentralem Venendruck und Volumenstatus eines Patienten, wie von Janssens u. Graf ebenfalls dort ausgeführt.

Intraabdominelle Druckerhöhungen haben in den letzten Jahren erheblich an Bedeutung gewonnen. Dies liegt am heutigen Routineverfahren Kapnoperitoneum, bei dem es als Folge der $\mathrm{CO}_{2}$-Insufflation $\mathrm{zu}$ erheblichen Implikationen für Beatmung und Hämodynamik kommen muss, wie Vogt u. Eberle im nächsten Heft beschreiben werden. Die auch daraus abzuleitende „Problematik der intraabdominellen Druckmessung “ erfordert nach Neumann (s. nächstes Heft) u. a. eine Vereinbarung über die Platzierung des Drucksensors, um den IAP lageunabhängig richtig messen zu können.

Unter dem Titel „Einfluss einer Volumenzunahme auf den intraabdominellen Druck“ wird von Schachtrupp (s. nächstes Heft) eindrucksvoll beschrieben werden, wie eine exzessive Volumentherapie mit Hyperhydratation zu einer pathologischen Erhöhung des IAP, dem sog. Kompartmentsyndrom, führen kann.

Bleibt zu hoffen, dass der Leser anhand dieser Beiträge erkennt, dass sich „Volumen macht Druck“ nicht nur für jedes Kompartiment allein, sondern auch im funktionell benachbarten Raum, positiv oder negativ auswirken kann.

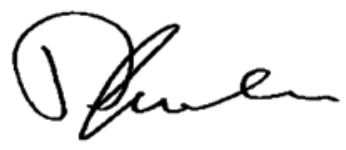

R. Zander

\section{Korrespondenzadresse \\ Prof. Dr. R. Zander \\ Physioklin \\ Luisenstraße 17, 55124 Mainz \\ zander@physioklin.de}

Interessenkonflikt. Der korrespondierende Autor gibt an, dass kein Interessenkonflikt besteht. 